TRANSACTIONS OF THE

AMERICAN MATHEMATICAL SOCIETY

Volume 362, Number 5, May 2010, Pages 2279-2299

S 0002-9947(09)04742-4

Article electronically published on December 14, 2009

\title{
DECAY ESTIMATES FOR WAVE EQUATIONS WITH VARIABLE COEFFICIENTS
}

\author{
PETRONELA RADU, GROZDENA TODOROVA, AND BORISLAV YORDANOV
}

\begin{abstract}
We establish weighted $L^{2}$-estimates for dissipative wave equations with variable coefficients that exhibit a dissipative term with a space dependent potential. These results yield decay estimates for the energy and the $L^{2}-$ norm of solutions. The proof is based on the multiplier method where multipliers are specially engineered from asymptotic profiles of related parabolic equations.
\end{abstract}

\section{INTRODUCTION}

Consider the following dissipative hyperbolic equation:

$$
u_{t t}-\operatorname{div}(b(x) \nabla u)+a(x) u_{t}=0, \quad x \in \mathbf{R}^{n}, \quad t>0,
$$

where the coefficients $a \in C^{0}(\mathbb{R}), b \in C^{1}(\mathbb{R})$ are positive functions, and the initial data $\left.u\right|_{t=0}=u_{0} \in H^{1}\left(\mathbb{R}^{n}\right),\left.u_{t}\right|_{t=0}=u_{1} \in L^{2}\left(\mathbb{R}^{n}\right)$ have compact support

$$
u_{0}(x)=0 \text { and } u_{1}(x)=0 \text { for }|x|>R \text {. }
$$

Such a system appears in models for traveling waves in a nonhomogeneous gas with damping that changes with the position. The unknown $u$ denotes the displacement, the coefficient $b$, called the bulk modulus, accounts for changes of the temperature depending on the location, while $a$ is referred to as the friction coefficient or potential (see [4 pp. 11-12 and 131). This problem has been studied intensively for the homogeneous medium, when $b$ is constant (see [11] for a review of the pertinent literature), but the results are scarce for the variable coefficient case. We first mention the work of Ikehata $[5$ in which the author finds rates of decay of solutions for a problem set on an exterior domain; however, no damping is present (Morawetz first looked at this problem with constant $b$ in [7]). In addition, Ikehata imposes that the bulk modulus be constant at infinity, so that $b$ is space dependent only inside a ball. In 3 S. J. Feng and D. X. Feng look at an equation with interior damping (possibly nonlinear) but for bounded domains. To our knowledge, the results of this paper are the first to be obtained for damped wave equations which exhibit space dependent hyperbolic operators and space dependent potential on the entire space $\mathbb{R}^{n}$.

Recently a strengthened multiplier method has been developed in [11] and used to derive the weighted energy of solutions for equations (1.1) with space dependent

Received by the editors October 4, 2007.

2000 Mathematics Subject Classification. Primary 35L05, 35L15; Secondary 37L15.

Key words and phrases. Wave equations with variable coefficients, linear dissipation, decay rates, subsolution. 
decaying potential $a(x)$ and $b=1$. For time dependent potentials $a(t)$ and $b(x)=1$ the Fourier transform yields optimal results; see [9, [10, [13, 14, 15].

The diffusion phenomenon for wave equations with damping (see [6], 8]) is crucial since it suggests considering multipliers related to a parabolic equation; these multipliers yield almost optimal decay estimates. Unfortunately the diffusion phenomenon for more general second-order hyperbolic equations with damping is not yet well understood.

The existence of a solution $u$ to (1.1) with the regularity

$$
u \in C\left((0, \infty), H^{1}\left(\mathbf{R}^{n}\right)\right), \quad u_{t} \in C\left((0, \infty), L^{2}\left(\mathbf{R}^{n}\right)\right),
$$

can be obtained through linear semigroup theory (see for example [1]).

We assume that

$$
b_{0}(1+|x|)^{\beta} \leq b(x) \leq b_{1}(1+|x|)^{\beta}
$$

and

$$
\frac{a_{0}}{(1+|x|)^{\alpha}} \leq a(x) \leq \frac{a_{1}}{(1+|x|)^{\alpha}}
$$

for some $\alpha, \beta \in \mathbb{R}$ and $a_{0}, a_{1}, b_{0}, b_{1}>0$.

This paper is an extensive generalization of 11 to the case of variable coefficients $b$. When $b$ is space dependent we find that although the general method of [11] remains viable, some nontrivial obstacles have to be surmounted. Since the problem does not have constant speed of propagation anymore, we have to construct new multipliers to obtain the desired estimates. A meticulous analysis also enables us to extend the range of exponents of [11] obtained for $b=1$. For a space dependent bulk modulus the results of this paper apply whenever $\alpha$ and $\beta$ belong to the following range of exponents:

$$
\alpha<1, \quad 0 \leq \beta<2, \quad 2 \alpha+\beta \leq 2 .
$$

Note that for $\alpha \leq 0$ and $0 \leq \beta<2$ the last inequality is always satisfied. In [1] the authors covered the case $0 \leq \alpha<1$ for $\beta=0$.

In the case of variable coefficient wave operators we observe some new phenomena. The decay rates pinpoint the interaction between the coefficients $a$ and $b$. Thus, an increase in the bulk modulus $b$ will trigger a faster decay of solutions, but one would have to decrease the damping coefficient $a$ to produce the same effect. Since the exponents that control the growth of $a$ and $b$ play opposite roles in the decay rates, we choose the asymmetric notation with $\alpha$ in the denominator and $\beta$ in the numerator in (1.3) and (1.2). It is worthwhile to mention that the energy decay rate goes to infinity (see Corollary 1.5) when $\beta \rightarrow 2^{-}$and $\alpha \rightarrow 0^{+}$. This shows that the range of the exponent $\beta$ in (1.4) is natural. On the other hand, in the case of overdamping, namely when $\alpha \rightarrow-\infty$ (see (1.3)), we prove that the energy decay stabilizes at $t^{-1}$.

In [1] the multiplier was constructed based on a solution to a related elliptic equation, but a more thorough analysis shows that a subsolution of the same elliptic equation is sufficient. This observation provides us with a better understanding of the problem so that we can ultimately find the natural multiplier for the equation. Also, we now have much more flexibility in constructing such functions (the set of subsolutions is considerably larger than the set of solutions). The far reaching applicability of our results is demonstrated in Section 7 where we explicitly find solutions and subsolutions to the elliptic problem in several broad cases; an exact 
solution was found in [11] only for the radial case. Our results also eliminate the radial restriction found from the main theorem in [1] (see the Remark after case (C3) in Section 7 for more details).

The results of this paper rely on the following

Hypothesis A. Under the above assumptions (1.2) and (1.3) there exists a subsolution $A(x)$ which satisfies

$$
\operatorname{div}(b(x) \nabla A(x)) \geq a(x), \quad x \in \mathbf{R}^{n},
$$

and has the following properties:

$$
\begin{array}{ll}
(a 1) & A(x) \geq 0 \text { for all } x, \\
(a 2) & A(x)=O\left(|x|^{2-\alpha-\beta}\right) \text { for large }|x|, \\
(a 3) & \mu:=\liminf _{x \rightarrow \infty} \frac{a(x) A(x)}{b(x)|\nabla A(x)|^{2}}>0 .
\end{array}
$$

The proof of the statement contained in Hypothesis A is straightforward in the radial case since it is a simple exercise to solve (1.5) with equality. We conjecture that the result holds true for arbitrary functions, since we were able to construct explicit solutions in many general situations. We had to impose only some mild restrictions in order to find an explicit subsolution $A$ that satisfies (1.5)-(1.8). A detailed discussion of all situations with which we can deal is included in Section 7.

Here we announce our main result.

Theorem 1.1. Assume that $a$ and $b$ satisfy (1.2) and (1.3) with $\alpha, \beta$ that satisfy (1.4), and assume that Hypothesis $A$ holds. Then for every $\delta>0$ the solution of (1.1) satisfies

$$
\begin{aligned}
& \int e^{(\mu-\delta) \frac{A(x)}{t}} a(x) u^{2} d x \leq C_{\delta}\left(\left\|\nabla u_{0}\right\|_{L^{2}}^{2}+\left\|u_{1}\right\|_{L^{2}}^{2}\right) t^{\delta-\mu}, \\
& \int e^{(\mu-\delta) \frac{A(x)}{t}}\left(u_{t}^{2}+b(x)|\nabla u|^{2}\right) d x \leq C_{\delta}\left(\left\|\nabla u_{0}\right\|_{L^{2}}^{2}+\left\|u_{1}\right\|_{L^{2}}^{2}\right) t^{\delta-\mu-1}
\end{aligned}
$$

for all $t \geq 1$. Here $A$ is the subsolution from Hypothesis $A$ and $\mu$ is the number defined by (1.8). The constant $C_{\delta}$ depends also on $R, a, b$, and $n$.

An important consequence that follows from the main theorem shows an exponential decay for the energy and the $L^{2}$-norm of the solution in the region $\left\{x: A(x) \geq t^{1+\varepsilon}\right\}$ with $\varepsilon>0$.

Corollary 1.2. Assume that conditions (1.2), (1.3), (1.4), and Hypothesis A hold. Then for every $\delta>0$ and $\varepsilon>0$ the solution of (1.1) satisfies

$$
\int_{A(x) \geq t^{1+\varepsilon}}\left(u^{2}+u_{t}^{2}+b(x)|\nabla u|^{2}\right) d x \leq C_{\delta}\left(\left\|\nabla u_{0}\right\|_{L^{2}}^{2}+\left\|u_{1}\right\|_{L^{2}}^{2}\right) e^{-(\mu-\delta) t^{\varepsilon}},
$$

for all $t \geq 1$, where $A$ and $\mu$ are given by Hypothesis $A$.

The fact that we are able to construct subsolutions in several cases (see Section 7) allows us to eliminate Hypothesis A from the assumptions. Thus we can formulate our main result in a more precise form that involves only the bounds for the coefficients $a$ and $b$ as in the following corollary. 
Corollary 1.3. Let $a$ and $b$ satisfy (1.2), (1.3), and (1.4). Then for each of the cases (C1)-(C4) listed in Section 7 there exists a constant $A_{0}>0$ such that

$$
\begin{aligned}
\int e^{A_{0}(\mu-\delta) \frac{|x|^{2-\alpha-\beta}}{t}} u^{2} d x & \leq C_{\delta}\left\{\begin{array}{l}
t^{\delta+\frac{\alpha}{2-\alpha-\beta}-\mu}, \quad \text { if } \alpha>0, \\
t^{\delta-\mu}, \quad \text { if } \alpha \leq 0,
\end{array}\right. \\
\int e^{A_{0}(\mu-\delta) \frac{|x|^{2-\alpha-\beta}}{t}}\left(u_{t}^{2}+b(x)|\nabla u|^{2}\right) d x & \leq C_{\delta} t^{\delta-\mu-1},
\end{aligned}
$$

where $t \geq 1$ and $\mu$ is given by (1.8) with $A$ computed in each of the cases (C1)-(C4).

Under some mild assumptions we can estimate $\mu$ in terms of the bounds for $a$ and $b$ alone. The following corollary states that under these circumstances the energy decays faster than $t^{\delta-\frac{a_{0} b_{0}}{a_{1} b_{1}} \cdot \frac{n-\alpha}{2-\alpha-\beta}-1}$, where $a_{0}, a_{1}, b_{0}, b_{1}$ are the constants from (1.2) and (1.3).

Corollary 1.4. If in addition to the assumptions of Theorem 1.1 we have $n \geq 2$, $\alpha<n-1$ and $b$ is such that

$$
\nabla b(x) \cdot \frac{x}{|x|} \geq b_{0} \beta(1+|x|)^{\beta-1}
$$

(these assumptions correspond to case (C4) in Section 7), then

$$
\begin{aligned}
& \int e^{(\mu-\delta) \frac{A(x)}{t}} a(x) u^{2} d x \leq C_{\delta}\left(\left\|\nabla u_{0}\right\|_{L^{2}}^{2}+\left\|u_{1}\right\|_{L^{2}}^{2}\right) t^{\delta-\mu}, \\
& \int e^{(\mu-\delta) \frac{A(x)}{t}}\left(u_{t}^{2}+b(x)|\nabla u|^{2}\right) d x \leq C_{\delta}\left(\left\|\nabla u_{0}\right\|_{L^{2}}^{2}+\left\|u_{1}\right\|_{L^{2}}^{2}\right) t^{\delta-\mu-1}
\end{aligned}
$$

for all $t \geq 1$, where a subsolution $A$ can be computed explicitly as in case (C4), and for $\mu$ defined by (1.8) we have the bound

$$
\mu \geq \frac{a_{0} b_{0}}{a_{1} b_{1}} \cdot \frac{n-\alpha}{2-\alpha-\beta} .
$$

The constant $C_{\delta}$ depends also on $R, a, b$ and $n$.

Remark 1. Note that (1.9) is always satisfied for functions $b$ which are radial. For arbitrary $b$ the condition (1.9) basically requires that the gradient of $b$ be larger than the gradient of the lower bound of $b$ given by (1.2). By choosing $b_{0}$ appropriately this condition can be satisfied by a large class of functions (including polynomials and rational functions).

Remark 2. If $a_{0}, a_{1}$, respectively $b_{0}, b_{1}$, are close to each other, then the decay for the energy is approximately of the order $t^{\delta-\frac{n-\alpha}{2-\alpha-\beta}-1}$. As we will see in the next corollary this is exactly the energy decay rate for $a$ and $b$ which behave like radial functions at infinity (obviously, this is the decay for $a$ and $b$ radially symmetric).

Corollary 1.5. Let a be an arbitrary (radial or not) function and $b$ be radial (these assumptions cover the cases (C1), (C3) in Section 7), or let a and b be both arbitrary but with the same angular component (as defined in Section 7 case (C2); see (7.3)). In addition, assume $n \geq 2$ and there exist $a_{2}, b_{2}>0$ such that

$$
a(x) \sim a_{2}|x|^{-\alpha}, \quad b(x) \sim b_{2}|x|^{\beta} \text { for large } x .
$$


Then, for every $\delta>0$ the solution of (1.1) satisfies

$$
\begin{aligned}
& \int e^{a_{2}(2-\alpha-\beta+\delta)^{-2} \frac{|x|^{2-\alpha-\beta}}{t}} u^{2} d x \\
& \leq C_{\delta}\left(\left\|\nabla u_{0}(x)\right\|_{L^{2}}^{2}+\left\|u_{1}(x)\right\|_{L^{2}}^{2}\right) \begin{cases}t^{\delta-\frac{n-2 \alpha}{2-\alpha-\beta}}, & \text { if } \alpha>0, \\
t^{\delta-\frac{n-\alpha}{2-\alpha-\beta}}, & \text { if } \alpha \leq 0,\end{cases} \\
& \left.\left.\int e^{a_{2}(2-\alpha-\beta+\delta)^{-2} \frac{|x|^{2-\alpha-\beta}}{t}}\left(u_{t}^{2}+b(x) \mid \nabla u\right)\right|^{2}\right) d x \\
& \leq C_{\delta}\left(\left\|\nabla u_{0}(x)\right\|_{L^{2}}^{2}+\left\|u_{1}(x)\right\|_{L^{2}}^{2}\right) t^{\delta-\frac{n-\alpha}{2-\alpha-\beta}-1} .
\end{aligned}
$$

2. Bounds on $A$ And $a$ ON The SUPport of the SOlution

Here we state a theorem about the support of solutions for a wave equation with variable coefficients. The proof closely follows the argument presented in [2], so we include a sketch.

Proposition 2.1 (Finite speed of propagation). Assume $u$ satisfies (1.1) and fix $x_{0} \in \mathbb{R}^{n}$ and $t_{0}>0$. Let $q(x)$ satisfy

$$
|\nabla q(x)|=b(x)^{-1 / 2}, \quad q\left(x_{0}\right)=0 .
$$

Consider the backward cone with vertex at $\left(x_{0}, t_{0}\right)$,

$$
C:=\left\{(x, t) \mid q(x)<t_{0}-t\right\},
$$

and its cross-section at time $t$,

$$
C_{t}:=\left\{x \mid q(x)<t_{0}-t\right\} .
$$

If $u(x, 0)=u_{t}(x, 0)=0$ on $C_{0}$, then $u(x, t)=0$ for $(x, t) \in C$.

Radial case. Assume $b(x)=b(|x|)$; then we have the following simplified statement:

If $u_{0}, u_{1}$ are supported inside the ball $|x|<R$, i.e.

$$
u_{0}(x)=u_{1}(x)=0 \text { for }|x|<R,
$$

then $u(x, t)=0$ whenever $|x|>R_{t}$, where

$$
R_{t}=q^{-1}(q(R)-t)
$$

By (2.1) the function $q$ is determined by

$$
q(r):=\left|\int_{r_{0}}^{r} b(s)^{-1 / 2} d s\right| .
$$

Sketch of the proof. By adjusting the proof of finite speed of propagation found in [2] (p. 395) to hyperbolic differential operators in nondivergence form, one obtains that $p$ solves the Hamilton-Jacobi equation

$$
p_{t}=\sqrt{b \sum_{i=1}^{n} p_{x_{i}}^{2}}=\sqrt{b|\nabla p|^{2}} .
$$

In order to solve for $p$ we separate the variables, so we write

$$
p=q+t-t_{0}
$$

where $q$ solves

$$
1=b(x)|\nabla q(x)|^{2},
$$


from which we obtain

$$
|\nabla q(x)|=(b(x))^{-1 / 2}
$$

From this point on the proof follows exactly the proof of Theorem 8 on p. 395 in [2].

Radial case. We solve $\left|q_{r}(r)\right|=(b(r))^{-1 / 2}$ and obtain

$$
q(r)= \pm \int_{r_{0}}^{r}(b(s))^{-1 / 2} d s, \quad \text { where } q\left(r_{0}\right)=0 .
$$

The cone of propagation is given by $q(r)=t_{0}-t$, and it has the vertex at $\left(r_{0}, t_{0}\right)$. Since $t_{0}>t$ we see that $q(r)$ must be nonnegative; in other words,

$$
q(r)= \begin{cases}\int_{r_{0}}^{r}(b(s))^{-1 / 2} d s, & \text { if } r_{0}<r \\ -\int_{r_{0}}^{r}(b(s))^{-1 / 2} d s, & \text { otherwise. }\end{cases}
$$

Consider the functions $u_{0}, u_{1}$ supported inside the ball of radius $R$. This implies that $u_{0}(r)=u_{1}(r)=0$ for $r>R$. Now consider the backward cone $\mathrm{C}$ given by $q(r)=t_{0}-t$ with vertex at $\left(r_{0}, t_{0}\right)$ which passes through the point $(R, 0)$. (The vertex depends on $R$.) This implies that $q(R)=t_{0}$. From the first part of the theorem we deduce that $u(r, t)=0$ for all points $(r, t)$ inside the backward cone; hence the support of $u$ is inside the forward cone $\mathrm{C}$, i.e.

$$
\text { supp } u \subset\left\{(r, t): q(r)>t_{0}-t\right\} \text {, with } r<r_{0} .
$$

In order to find the size of the support at time $t$ we need to find the $r$ coordinate (denoted by $R_{t}$ ) of the point on the forward cone at time $t$. We have

$$
q\left(R_{t}\right)=t_{0}-t=q(R)-t
$$

hence $R_{t}=q^{-1}(q(R)-t)$. Since we only looked at the branch for $q$ corresponding to $r<r_{0}$, we have that $q$ is invertible (this branch is a strictly increasing function).

For the radial case a couple of observations are in order. First note that $q(R)$ is well defined even if $r_{0}=0$, since $b(r)^{-1 / 2}$ is integrable at the origin (this follows from (1.2)). Second, one can easily prove that $R_{t}$ does not actually depend on the choice for the vertex of the cone. If one were to choose the vertex for a different $r_{0}$ (let us denote it by $\bar{r}_{0}$ ), then the function $q$ would change to some $\bar{q}$ with $\bar{q}\left(\bar{r}_{0}\right)=0$. Since $q_{r}=\bar{q}_{r}$ one obtains that

$$
R_{t}=q^{-1}(q(R)-t)=\bar{q}^{-1}(\bar{q}(R)-t) .
$$

The formula (2.2) is not very useful in this form, since we cannot directly see how fast or slow the support of the solution grows. For this reason we will be interested in finding some bounds for $R_{t}$ using the growth conditions on $b$. Take $b(x)=b_{1}(1+|x|)^{\beta}$, so for $r<r_{0}$ we have

$$
q(r)=-\int_{r_{0}}^{r} \frac{1}{\sqrt{(1+s)^{\beta}}} d s .
$$

Since $\beta<2$, we find

$$
q(r)=\frac{1}{\sqrt{b_{1}}}\left[\left(1+r_{0}\right)^{(2-\beta) / 2}-(1+r)^{(2-\beta) / 2}\right] .
$$


Hence $q^{-1}(y)=\left[\left(1+r_{0}\right)^{(2-\beta) / 2}-y \sqrt{b_{1}}\right]^{2 /(2-\beta)}-1$ and

$$
R_{t}=\left[(1+R)^{(2-\beta) / 2}+t \sqrt{b_{1}}\right]^{2 /(2-\beta)} .
$$

Note that for a general $b$ satisfying (1.2) with $\beta<2$, the above $R_{t}$ will provide an upper bound. One has that the radius $R_{t}$ for a general $b$ satisfies the following estimate:

$$
R_{t} \sim R+C t^{(2-\beta) / 2} .
$$

Remark. These results can also be deduced from Theorem 2.4 and Theorem 2.7 (pp. 45-47) in 44) regarding the finite speed of propagation and the domain of influence for solutions of boundary value problems associated with hyperbolic differential equations with variable coefficients in nondivergence form.

Proposition 2.2. Define

$$
\begin{aligned}
g(t) & :=\inf \{a(x): x \in \operatorname{supp} u(\cdot, t)\}, \\
G(t) & :=\sup \{A(x): x \in \operatorname{supp} u(\cdot, t)\},
\end{aligned}
$$

and

$$
\gamma=\left\{\begin{array}{l}
\frac{2 \alpha}{2-\beta}, \quad \text { if } \beta \leq 2-2 \alpha, \\
0, \quad \text { if } \alpha \leq 0
\end{array}\right.
$$

Then

$$
\begin{gathered}
g(t) \geq g_{0} t^{-\gamma}, \quad t \geq T, \\
G(t) \leq G_{0} t^{2-\gamma}, \quad t \geq T,
\end{gathered}
$$

where $g_{0}$ and $G_{0}$ are positive constants.

Proof. From (2.3), the support of $u$ is contained in the set

$$
|x| \leq\left[(1+R)^{(2-\beta) / 2}+t \sqrt{b_{0}}\right]^{2 /(2-\beta)} .
$$

Then we can take the function

$$
g(t)= \begin{cases}a_{0}\left[R+C t^{2 /(2-\beta)}\right]^{-\alpha}, & \text { if } \alpha \geq 0, \\ a_{0}, & \text { if } \alpha<0 .\end{cases}
$$

To verify the upper bound on $G(t)$ we just use $A(x)=O\left(|x|^{2-\alpha-\beta}\right)$ for large $|x|$.

\section{A WEIGHTED ENERGY IDENTITY}

We consider dissipative hyperbolic equations of the form

$$
u_{t t}-\operatorname{div}(b(x) \nabla u)+a(x) u_{t}=0,
$$

where the coefficients $a$ and $b$ are $C^{1}$-functions. Our goal is to derive a weighted energy identity for $u$ involving three positive $C^{2}$-functions: $\eta, \theta$ and $\varphi$.

Let $\hat{u}=\varphi^{-1} u$ and substitute $u=\varphi \hat{u}$ into equation (3.1). We have that

$$
\hat{u}_{t t}-\hat{b}_{1} \Delta \hat{u}-\hat{b}_{2} \cdot \nabla \hat{u}+\hat{a}_{1} \hat{u}_{t}+\hat{a}_{2} \hat{u}=0,
$$


where the new coefficients are

$$
\begin{aligned}
& \hat{b}_{1}=b, \quad \hat{b}_{2}=\nabla b+2 b \varphi^{-1} \nabla \varphi \\
& \hat{a}_{1}=a+2 \varphi^{-1} \varphi_{t}, \quad \hat{a}_{2}=\varphi^{-1}\left(\varphi_{t t}-\operatorname{div}(b \nabla \varphi)+a \varphi_{t}\right) .
\end{aligned}
$$

We multiply the equation for $\hat{u}$ with $\eta \hat{u}+\theta \hat{u}_{t}$ and integrate on $\mathbf{R}^{n}$ using the divergence theorem. The boundary terms vanish since $\hat{u}(x, t)$ has compact support with respect to $x$. Details are given in Appendix A.

Proposition 3.1. Let $u$ be a solution of (3.1). Assume that $\eta, \theta$, and $\varphi>0$ are $C^{2}$-functions. Then $\hat{u}$ satisfies

$$
\frac{d}{d t} E\left(\hat{u}_{t}, \nabla \hat{u}, \hat{u}\right)+F\left(\hat{u}_{t}, \nabla \hat{u}\right)+G(\hat{u}, \nabla \hat{u})=0,
$$

where

$$
\begin{aligned}
E\left(\hat{u}_{t}, \nabla \hat{u}, \hat{u}\right)= & \frac{1}{2} \int\left[\theta\left(\hat{u}_{t}^{2}+b_{1}|\nabla \hat{u}|^{2}\right)+2 \eta \hat{u}_{t} \hat{u}+\left(\hat{a}_{2} \theta-\eta_{t}+\hat{a}_{1} \eta\right) \hat{u}^{2}\right] d x \\
F\left(\hat{u}_{t}, \nabla \hat{u}\right)= & \frac{1}{2} \int\left(-\theta_{t}+2 \hat{a}_{1} \theta-2 \eta\right) \hat{u}_{t}^{2} d x \\
& +\int\left(\nabla\left(\theta \hat{b}_{1}\right)-\theta \hat{b}_{2}\right) \cdot \hat{u}_{t} \nabla \hat{u} d x \\
& +\frac{1}{2} \int\left(-\left(\hat{b}_{1} \theta\right)_{t}+2 \hat{b}_{1} \eta\right)|\nabla \hat{u}|^{2} d x \\
G(\hat{u})= & \frac{1}{2} \int\left[\eta_{t t}-\left(\hat{a}_{1} \eta\right)_{t}+\operatorname{div}\left(\eta \hat{b}_{2}-\nabla\left(\eta \hat{b}_{1}\right)\right)+2 \hat{a}_{2} \eta-\left(\hat{a}_{2} \theta\right)_{t}\right] \hat{u}^{2} d x
\end{aligned}
$$

The coefficients $\hat{b}_{1}, \hat{b}_{2}, \hat{a}_{1}$ and $\hat{a}_{2}$ are defined in (3.3).

\section{Hyperbolic equations With VARIABle DAMPing}

We will apply Proposition 3.1 to the hyperbolic equation with damping (1.1). The main difficulty is to find three weights that can yield sharp decay estimates for $u$. A good choice is $\eta=\varphi$, since it simplifies the weighted identity for $\hat{u}$. It is not clear how $\theta$ depends on $\varphi$, so this choice is postponed.

Below we restate the identity in Proposition 3.1. We express all coefficients except $\hat{a}_{2}$ in terms of $\varphi, \theta, a$ and $b$. The most significant differences appear in $G(\hat{u})$. We obtain

$$
\begin{aligned}
E\left(\hat{u}_{t}, \nabla \hat{u}, \hat{u}\right)= & \frac{1}{2} \int\left[\theta\left(\hat{u}_{t}^{2}+b|\nabla \hat{u}|^{2}\right)+2 \varphi \hat{u}_{t} \hat{u}+\left(\hat{a}_{2} \theta+\varphi_{t}+a \varphi\right) \hat{u}^{2}\right] d x \\
F\left(\hat{u}_{t}, \nabla \hat{u}\right)= & \frac{1}{2} \int\left(-\theta_{t}+2\left(a+2 \varphi^{-1} \varphi_{t}\right) \theta-2 \varphi\right) \hat{u}_{t}^{2} d x \\
& +\int b\left(\nabla \theta-2 \theta \varphi^{-1} \nabla \varphi\right) \cdot \hat{u}_{t} \nabla \hat{u} d x \\
& +\frac{1}{2} \int b\left(-\theta_{t}+2 \varphi\right)|\nabla \hat{u}|^{2} d x \\
G(\hat{u})= & \frac{1}{2} \int\left[\hat{a}_{2} \varphi-\left(\hat{a}_{2} \theta\right)_{t}\right] \hat{u}^{2} d x
\end{aligned}
$$


and the following identity:

$$
\frac{d}{d t} E\left(\hat{u}_{t}, \nabla \hat{u}, \hat{u}\right)+F\left(\hat{u}_{t}, \nabla \hat{u}\right)+G(\hat{u})=0 .
$$

The next step is to show that $F \geq 0$ and $G \geq 0$ under certain conditions on the weights and coefficients. Hence $d E / d t \leq 0$, which implies that the weighted energy is a decreasing function of $t$.

Proposition 4.1. Let $\varphi$ and $\theta$ be positive weights, such that

$$
\begin{aligned}
& \hat{a}_{2} \geq 0, \quad\left(\hat{a}_{2}\right)_{t} \leq 0, \\
& -\theta_{t}+\varphi \geq 0, \\
& \left(-\theta_{t}+2\left(a+2 \varphi^{-1} \varphi_{t}\right) \theta-2 \varphi\right)\left(-\theta_{t}+2 \varphi\right) \geq b\left(\nabla \theta-2 \theta \varphi^{-1} \nabla \varphi\right)^{2} .
\end{aligned}
$$

Then $E$ is a nonincreasing function: $d E\left(\hat{u}_{t}, \nabla \hat{u}, \hat{u}\right) / d t \leq 0$ and

$$
\frac{1}{2} \int\left[\theta\left(\hat{u}_{t}^{2}+b|\nabla \hat{u}|^{2}\right)+2 \varphi \hat{u}_{t} \hat{u}+\left(\hat{a}_{2} \theta+\varphi_{t}+a \varphi\right) \hat{u}^{2}\right] d x \leq E_{0}
$$

for all $t \geq T$, where $E_{0}=\left.E\left(\hat{u}_{t}, \nabla \hat{u}, \hat{u}\right)\right|_{t=T}$.

Proof. To see that $G(\hat{u}) \geq 0$, we combine $(i),(i i)$ :

$$
\hat{a}_{2} \varphi-\left(\hat{a}_{2} \theta\right)_{t}=\hat{a}_{2}\left(\varphi-\theta_{t}\right)-\left(\hat{a}_{2}\right)_{t} \theta \geq 0 .
$$

Using conditions $\left(\right.$ iii) and $-\theta_{t}+2 \varphi \geq 0$, which follows from $($ ii), we obtain that the quadratic form $F\left(\hat{u}_{t}, \nabla \hat{u}\right) \geq 0$. Hence (4.1) implies $d E\left(\hat{u}_{t}, \nabla \hat{u}, \hat{u}\right) / d t \leq 0$.

Clearly $E\left(\hat{u}_{t}, \nabla \hat{u}, \hat{u}\right) \leq E_{0}$ is a useful estimate only for positive definite quadratic forms of $\hat{u}_{t}, \nabla \hat{u}$ and $\hat{u}$. To guarantee this condition we derive a preliminary estimate of $\hat{u}$. Notice that neglecting the positive terms $\theta\left(\hat{u}_{t}^{2}+b|\nabla \hat{u}|^{2}\right)$ and $\hat{a}_{2} \theta \hat{u}^{2}$ in Proposition 4.1 yields the inequality

$$
\frac{1}{2} \frac{d}{d t} \int \varphi \hat{u}^{2} d x+\frac{1}{2} \int a \varphi \hat{u}^{2} d x \leq E_{0} .
$$

This is the starting point to bound the $L^{2}$-norm of $\hat{u}$ if $a$ satisfies (1.3).

In order to prove our next proposition we will need the following

Lemma 4.2. Let $g \in C([T, \infty))$ be a positive function. Then

$$
e^{-\int_{T}^{t} g(s) d s} \int_{T}^{t} e^{\int_{T}^{s} g(\tau) d \tau} d s \leq \max _{s \in[T, t]} \frac{1}{g(s)} .
$$

Proof. Compute

$$
\begin{aligned}
\int_{T}^{t} e^{\int_{T}^{s} g(\tau) d \tau} d s & \leq\left[\max _{s \in[T, t]} \frac{1}{g(s)}\right] \int_{T}^{t} g(s) e^{\int_{T}^{s} g(\tau) d \tau} d s \\
& =\left[\max _{s \in[T, t]} \frac{1}{g(s)}\right]\left[e^{\int_{T}^{s} g(\tau) d \tau}\right]_{T}^{t} \leq\left[\max _{s \in[T, t]} \frac{1}{g(s)}\right] e^{\int_{T}^{t} g(s) d s} .
\end{aligned}
$$

The last inequality completes the proof.

Proposition 4.3. Let $\varphi$ and $\theta$ be positive weights satisfying conditions (i)-(iii) in Proposition 4.1. Assume that (1.3) holds. Then

$$
\int \varphi \hat{u}^{2} d x \leq H_{0}+C E_{0} t^{\gamma}, \quad t \geq T
$$


where

$$
H_{0}=\left.\int \varphi \hat{u}^{2} d x\right|_{t=T}
$$

and $\gamma$ is the exponent defined in (2.6).

Proof. From (4.2) we have

$$
\frac{d}{d t} \int \varphi \hat{u}^{2} d x+g(t) \int \varphi \hat{u}^{2} d x \leq 2 E_{0}
$$

where $g$ was defined in (2.4).

The above inequality implies

$$
\int \varphi \hat{u}^{2} d x \leq e^{-\int_{T}^{t} g(s) d s}\left[H_{0}+2 E_{0} \int_{T}^{t} e^{\int_{T}^{s} g(\tau) d \tau} d s\right], \quad t \geq T .
$$

By Lemma 4.2 and the fact that $g$ is decreasing, we obtain

$$
e^{-\int_{T}^{t} g(s) d s} \int_{T}^{t} e^{\int_{T}^{s} g(\tau) d \tau} d s \leq \max _{s \in[T, t]} \frac{1}{g(s)} \leq \frac{1}{g(t)}
$$

The following inequality and (2.6) yield the claimed estimate:

$$
\int \varphi \hat{u}^{2} d x \leq H_{0}+\frac{E_{0}}{g(t)} .
$$

Proposition 4.4. Assume that a satisfies (1.3). If $\varphi$ and $\beta$ satisfy conditions (i)-(iii) in Proposition 4.1 and the additional conditions

$$
\begin{aligned}
\text { (iv) } & \varphi \leq C_{0} t^{-\gamma} \theta, \\
\text { (v) } & \varphi_{t} \geq-C_{0} t^{-\gamma} \varphi
\end{aligned}
$$

with $C_{0}>0$, then

$$
\begin{aligned}
\int \theta\left(\hat{u}_{t}^{2}+b|\nabla \hat{u}|^{2}\right) d x & \leq C\left(H_{0}+E_{0}\right), \\
\int a \varphi \hat{u}^{2} d x & \leq C\left(H_{0}+E_{0}\right),
\end{aligned}
$$

for $t \geq T$.

Proof. Recall the inequality for $E$ derived in Proposition 4.1

$$
\frac{1}{2} \int\left[\theta\left(\hat{u}_{t}^{2}+b|\nabla \hat{u}|^{2}\right)+2 \varphi \hat{u}_{t} \hat{u}+\left(\hat{a}_{2} \theta+\varphi_{t}+a \varphi\right) \hat{u}^{2}\right] d x \leq E_{0}
$$

for all $t \geq T$, where $E_{0}=\left.E\left(\hat{u}_{t}, \nabla \hat{u}, \hat{u}\right)\right|_{t=T}$. Using

$$
\left|2 \varphi \hat{u}_{t} \hat{u}\right| \leq \varepsilon t^{\gamma} \varphi \hat{u}_{t}^{2}+\varepsilon^{-1} t^{-\gamma} \varphi \hat{u}^{2}, \quad \varepsilon \in(0,1),
$$

we obtain

$$
\int\left[\left(\theta-\varepsilon t^{\gamma} \varphi\right)\left(\hat{u}_{t}^{2}+b|\nabla \hat{u}|^{2}\right)+a \varphi \hat{u}^{2}\right] d x \leq 2 E_{0}+\int\left(\varepsilon^{-1} t^{-\gamma} \varphi-\hat{a}_{2} \theta-\varphi_{t}\right) \hat{u}^{2} d x .
$$

To complete the proof we choose $\varepsilon=\left(2 C_{0}\right)^{-1}$ and apply Proposition 4.3 . 
Proposition 4.5. Assume (1.2), (1.3), (i) $-(v)$ and

$$
\text { (vi) } \quad \theta \varphi^{-3}\left(\varphi_{t}^{2}+b|\nabla \varphi|^{2}\right) \leq C_{0} a
$$

for some $C_{0}>0$. Then the estimates

$$
\begin{aligned}
\int a \varphi^{-1} u^{2} d x & \leq C\left(H_{0}+E_{0}\right), \\
\int \theta \varphi^{-2}\left(u_{t}^{2}+b|\nabla u|^{2}\right) d x & \leq C\left(H_{0}+E_{0}\right)
\end{aligned}
$$

hold for $t \geq t_{0}$.

Proof. In terms of $u$, Proposition 4.3 and Proposition 4.4 can be stated as

$$
\begin{aligned}
& \int \varphi^{-1} u^{2} d x \leq H_{0}+C E_{0} t^{\gamma}, \\
& \int a \varphi^{-1} u^{2} d x \leq C\left(H_{0}+E_{0}\right),
\end{aligned}
$$

respectively. We need another assumption on $\varphi$ and $\theta$ in order to derive similar estimates for $u_{t}$ and $\nabla u$. Note that

$$
\hat{u}_{t}^{2}=\left(-\varphi^{-2} \varphi_{t} u+\varphi^{-1} u_{t}\right)^{2} \geq \frac{1}{2} \varphi^{-2} u_{t}^{2}-\varphi^{-4} \varphi_{t}^{2} u^{2}
$$

and

$$
|\nabla \hat{u}|^{2}=\left(-\varphi^{-2} \nabla \varphi u+\varphi^{-1} \nabla u\right)^{2} \geq \frac{1}{2} \varphi^{-2}|\nabla u|^{2}-\varphi^{-4}|\nabla \varphi|^{2} u^{2} .
$$

Combined with the estimate in Proposition 4.4, these inequalities imply

$$
\frac{1}{2} \int \theta \varphi^{-2}\left(u_{t}^{2}+b|\nabla u|^{2}\right) d x \leq C\left(H_{0}+E_{0}\right)+\int \theta \varphi^{-4}\left(\varphi_{t}^{2}+b|\nabla \varphi|^{2}\right) u^{2} d x .
$$

If the weights satisfy $\theta \varphi^{-4}\left(\varphi_{t}^{2}+b|\nabla \varphi|^{2}\right) \leq C_{0} a \varphi^{-1}$, we can apply Proposition 4.3 to obtain the final estimates.

\section{EXISTENCE OF $\varphi$ AND $\theta$}

There are nontrivial weights $\varphi$ and $\theta$ satisfying all conditions $(i)-(v i)$ in the previous section. Actually we can choose $\varphi$ to be an approximate solution of (1.1) and $\theta$ that behaves like a time integral of $\varphi$. This approach uses solutions (or subsolutions; see the Remark below) of the elliptic equation in divergence form

$$
\operatorname{div}(b(x) \nabla A(x))=a(x), \quad x \in \mathbf{R}^{n} .
$$

We assume that there exist such $A$ with properties $(a 1)-(a 3)$ from the Introduction. To define the weights $\varphi$ and $\theta$, we also choose $\sigma_{0}>0$ and $\delta \in\left(0, \frac{1}{2} \mu\right)$. Let

$$
m=\mu-2 \delta, \quad \sigma(x)=(\mu-\delta) A(x)+\sigma_{0} .
$$

Then the two weights are given by

$$
\varphi(x, t)=t^{-m} e^{-\frac{\sigma(x)}{t}}, \quad \theta(x, t)=\frac{3}{4}\left(\frac{6}{t}+\frac{\sigma(x)}{t^{2}}\right)^{-1} \varphi(x, t) .
$$

We should mention that the parameters $\sigma_{0}, \delta, \frac{3}{4}$ and 6 are introduced for technical reasons. It is clear that $\mu$ gives the $L^{2}$-decay rate. The presence of $\delta>0$ in our estimates leads to the loss of decay $t^{-\delta}$. We can probably avoid this parameter if we allow logarithmic terms. Concerning the role of $\sigma(x)$, we will see that it determines 
the actual support of solutions. In particular, the solutions decay fast in the region where $A(x) / t \rightarrow \infty$.

For convenience we restate conditions $(a 1)-(a 3)$ in terms of $\mu$ and $\sigma(x)$ :

$(\sigma 1) \quad \operatorname{div}(b(x) \nabla \sigma(x)) \geq(m+\delta) a(x)$ for all $x$,

$(\sigma 2) \quad \sigma(x)=O\left(|x|^{2-\alpha-\beta}\right)$ for large $|x|$,

$$
\left(1-\frac{\delta}{2 \mu}\right) a(x) \sigma(x)-b(x)|\nabla \sigma(x)|^{2} \geq 0 \text { for all } x .
$$

(Introducing large $\sigma_{0}$ ensures condition $(\sigma 3)$ for all $x$.)

Remark. Note that it is enough to find a subsolution to the elliptic equation, i.e. a function $A$ such that

$$
\operatorname{div}(b(x) \nabla A(x)) \geq a(x)
$$

that satisfies (a1)-(a3). This is due to the fact that $(\sigma 1)-(\sigma 3)$ hold for $\sigma$ as chosen in (5.2).

The rest of this section verifies conditions $(i)-(v i)$ for the weights $\varphi$ and $\theta$ defined in (5.3). We begin with the following useful calculations:

$$
\begin{aligned}
\varphi_{t} & =\left(-\frac{m}{t}+\frac{\sigma(x)}{t^{2}}\right) \varphi, \quad \varphi_{t t}=\left(-\frac{m}{t}+\frac{\sigma(x)}{t^{2}}\right)^{2} \varphi+\left(\frac{m}{t^{2}}-\frac{2 \sigma(x)}{t^{3}}\right) \varphi, \\
\nabla \varphi & =-\frac{\nabla \sigma(x)}{t} \varphi, \quad \operatorname{div}(b(x) \nabla \varphi)=\frac{\varphi}{t^{2}} b(x)|\nabla \sigma(x)|^{2}-\frac{\varphi}{t} \operatorname{div}(b(x) \nabla \sigma(x))
\end{aligned}
$$

and

$$
\frac{\theta_{t}}{\theta}=-\frac{m}{t}+\frac{\sigma(x)}{t^{2}}+\left(\frac{6}{t}+\frac{2 \sigma(x)}{t^{2}}\right)\left(6+\frac{\sigma(x)}{t}\right)^{-1} .
$$

We use (5.5) to calculate the coefficient $\hat{a}_{2}$ in (3.3):

$$
\begin{aligned}
\hat{a}_{2}= & \frac{\operatorname{div}(b(x) \nabla \sigma(x))-m a(x)}{t}+\frac{a(x) \sigma(x)-b(x)|\nabla \sigma(x)|^{2}}{t^{2}} \\
& +\left(-\frac{m}{t}+\frac{\sigma(x)}{t^{2}}\right)^{2}+\frac{m}{t^{2}}-\frac{2 \sigma(x)}{t^{3}} .
\end{aligned}
$$

Another simple observation is that (5.6) implies

$$
\frac{\theta_{t}}{\theta} \leq \frac{-m+1}{t}+\frac{4}{3} \frac{\sigma(x)}{t^{2}}
$$

The next result confirms our choice of $\mu$ and $\sigma(x)$.

Proposition 5.1. Let $\varphi$ and $\theta$ be defined in (5.3). Then conditions (i)-(vi) on $\varphi$ and $\theta$ hold for sufficiently large $t \geq T$.

Proof. ( $i$ ) Equality (5.7) and conditions $(\sigma 1),(\sigma 3)$ yield

$$
\begin{aligned}
\hat{a}_{2} & \geq \frac{\delta a(x)}{t}+\frac{\varepsilon a(x) \sigma(x)}{t^{2}}-\frac{2 \sigma(x)}{t^{3}} \\
& =\frac{\delta a(x)}{t}+\frac{\sigma(x)\left(\varepsilon a(x)-2 t^{-1}\right)}{t^{2}},
\end{aligned}
$$

where $\varepsilon=\delta /(2 \mu)$. Since $g(t) \geq g_{0} t^{-\gamma}$ and $\gamma<1$, the second term on the right hand side is positive for sufficiently large $t$. Hence $\hat{a}_{2} \geq 0$. The proof that $\left(\hat{a}_{2}\right)_{t}<0$ is similar. 
(ii) It follows from definitions (5.3) and inequality (5.8) that

$$
\begin{aligned}
-\theta_{t}+\varphi & =\left(-\frac{\theta_{t}}{\theta}+\frac{\varphi}{\theta}\right) \theta \\
& \geq\left(\frac{m-1}{t}-\frac{4}{3} \frac{\sigma(x)}{t^{2}}+\frac{8}{t}+\frac{4}{3} \frac{\sigma(x)}{t^{2}}\right) \theta \\
& \geq 0 .
\end{aligned}
$$

(iii) This inequality contains two factors on the left side. The first is estimated by the previous inequality $(i i)$ :

$$
\begin{aligned}
-\theta_{t}+2 \varphi & =\left(-\theta_{t}+\varphi\right)+\varphi \\
& \geq \varphi
\end{aligned}
$$

Unfortunately, the second factor requires more involved calculations:

$$
\begin{aligned}
-\theta_{t}+2\left(a+2 \varphi^{-1} \varphi_{t}\right) \theta-2 \varphi= & \left(-\frac{\theta_{t}}{\theta}+2 a+4 \frac{\varphi_{t}}{\varphi}-2 \frac{\varphi}{\theta}\right) \theta \\
\geq & \left(\frac{m-1}{t}-\frac{4}{3} \frac{\sigma(x)}{t^{2}}+2 a(x)\right) \theta \\
& +\left(-\frac{4 m}{t}+\frac{4 \sigma(x)}{t^{2}}-\frac{16}{t}-\frac{8}{3} \frac{\sigma(x)}{t^{2}}\right) \theta .
\end{aligned}
$$

We can regroup the terms to obtain

$$
\begin{aligned}
-\theta_{t}+2\left(a+2 \varphi^{-1} \varphi_{t}\right) \theta-2 \varphi & \geq\left(-\frac{3 m+17}{t}+2 a(x)\right) \theta \\
& \geq a(x) \theta
\end{aligned}
$$

for sufficiently large $t$. This follows from the fact that for large $t$,

$$
a(x) \geq g_{0} t^{-\gamma} \text { with } \gamma<1, \quad(x, t) \in \operatorname{supp} u,
$$

as it was proven in Proposition 2.2,

Multiplying the two lower bounds,

$$
\begin{aligned}
\left(-\theta_{t}+2 \varphi\right)\left(-\theta_{t}+2 a \theta+4 \theta \varphi^{-1} \varphi_{t}-2 \varphi\right) & \geq \varphi \cdot a \theta \\
& =\frac{4 a(x)(6 t+\sigma(x))}{3 t^{2}} \theta^{2} \\
& \geq \frac{4}{3} \frac{a(x) \sigma(x)}{t^{2}} \theta^{2} .
\end{aligned}
$$

It remains to find the expression on the right side of $(i i i)$ :

$$
\begin{aligned}
\nabla \theta-2 \theta \varphi^{-1} \nabla \varphi & =\left(\frac{\nabla \theta}{\theta}-2 \frac{\nabla \varphi}{\varphi}\right) \theta \\
& =\left(-\frac{\nabla \sigma(x)}{6 t+\sigma(x)}+\frac{\nabla \sigma(x)}{t}\right) \theta \\
& =\frac{(5 t+\sigma(x)) \nabla \sigma(x)}{t(6 t+\sigma(x))} \theta .
\end{aligned}
$$

Hence

$$
b(x)\left(\nabla \theta-2 \theta \varphi^{-1} \nabla \varphi\right)^{2}=b(x) \frac{(5 t+\sigma(x))^{2}|\nabla \sigma(x)|^{2}}{t^{2}(6 t+\sigma(x))^{2}} \theta^{2} .
$$


Condition $(\sigma 3)$ shows that $b(x)|\nabla \sigma(x)|^{2} \leq a(x) \sigma(x)$, so the above expression can be compared with the term

$$
\frac{4}{3} \frac{a(x) \sigma(x)}{t^{2}} \theta^{2}
$$

from (15.9):

$$
\frac{(5 t+\sigma(x))^{2} b(x)|\nabla \sigma(x)|^{2}}{t^{2}(6 t+\sigma(x))^{2}} \theta^{2} \leq \frac{a(x) \sigma(x)(5 t+\sigma(x))^{2}}{t^{2}(6 t+\sigma(x))^{2}} \theta^{2} \leq \frac{a(x) \sigma(x)}{t^{2}} \theta^{2} .
$$

Hence condition ( $i i i)$ holds.

$(i v)$ From $(\sigma 2)$, we have that

$$
\frac{\varphi(t, x)}{\theta(t, x)}=\frac{4}{3} \frac{6 t+\sigma(x)}{t^{2}}
$$

Hence the right hand side in the above inequality is bounded by $C t^{-\gamma}$ by (2.8).

$(v)$ Since $\gamma<1$, this condition is trivially satisfied.

(vi) An equivalent condition is

$$
\left(m+\frac{\sigma(x)}{t}\right)^{2}+b(x)|\nabla \sigma(x)|^{2} \leq C(6 t+\sigma(x)) a(x) .
$$

First note that since $b(x)|\nabla \sigma(x)| \leq a(x) \sigma(x)$, it is enough to show that

$$
\left(m+\frac{\sigma(x)}{t}\right)^{2} \leq C(t+\sigma(x)) a(x) .
$$

This is satisfied since $m^{2} \leq C a(x) t$ and $\frac{\sigma(x)}{t^{2}} \leq C a(x)$ by (2.7) and (2.8).

\section{Proofs of Theorem 1.1 And Corollaries 1.2 And 1.3}

According to Proposition 5.1, $\varphi$ and $\theta$ are admissible weights for the decay estimates in Proposition 4.5. We can now derive the main result and its two corollaries.

Proof of Theorem 1.1. Since the estimates are trivial for small $t \geq 1$, we can assume that $t \geq T$ and $T$ is sufficiently large. We will apply Proposition 4.5 with the weights defined in (5.2) and (5.3). For every $\delta>0$, we obtain

$$
\begin{aligned}
\int e^{(\mu-\delta) \frac{A(x)}{t}} a(x) u^{2} d x & \leq C\left(H_{0}+E_{0}\right) t^{2 \delta-\mu}, \\
\int e^{(\mu-\delta) \frac{A(x)}{t}}\left(\frac{1}{t}+\frac{A(x)}{t^{2}}\right)^{-1}\left(u_{t}^{2}+b(x)|\nabla u|^{2}\right) d x & \leq C\left(H_{0}+E_{0}\right) t^{2 \delta-\mu},
\end{aligned}
$$

where $t \geq T$. We can rewrite the second estimate using

$$
\left(\frac{1}{t}+\frac{A(x)}{t^{2}}\right)^{-1} t\left(1+\frac{A(x)}{t}\right)^{-1} \geq C_{0} t e^{-\delta \frac{A(x)}{t}},
$$

with some $C_{0}>0$ depending on $\delta$ and $T$. Notice also that

$$
H_{0}+E_{0} \leq C_{1}\left(\left\|\nabla u_{0}\right\|_{L^{2}}^{2}+\left\|u_{1}\right\|_{L^{2}}^{2}\right)
$$

where $C_{1}$ depends on $R, a, b$, and $n$. This yields the estimates in Theorem 1.1 with a loss of decay $2 \delta$. To obtain the final form we only replace $2 \delta$ by $\delta$. 
Proof of Corollary 1.2. Adding the two estimates in Theorem 1.1 and restricting the integration to $\left\{x: A(x) \geq t^{1+\varepsilon}\right\}$, we have

$$
\int_{A(x) \geq t^{1+\varepsilon}} e^{(\mu-\delta) \frac{A(x)}{t}}\left(a u^{2}+u_{t}^{2}+b|\nabla u|^{2}\right) d x \leq C_{\delta}\left(\left\|\nabla u_{0}\right\|_{L^{2}}^{2}+\left\|u_{1}\right\|_{L^{2}}^{2}\right) t^{\delta-\mu} .
$$

Since $A(x) / t \geq t^{\varepsilon}$ and $a(x) \geq g_{0} t^{-\gamma}$, by Proposition 2.2, this inequality implies

$$
\int_{A(x) \geq t^{1+\varepsilon}}\left(u^{2}+u_{t}^{2}+b|\nabla u|^{2}\right) d x \leq C_{\delta}\left(\left\|\nabla u_{0}\right\|_{L^{2}}^{2}+\left\|u_{1}\right\|_{L^{2}}^{2}\right) t^{\delta+\gamma-\mu} e^{-(\mu-\delta) t^{\varepsilon}} .
$$

The power of $t$ can be accounted for by a slight increase of $\delta$.

Proof of Corollary 1.3. To obtain these weighted estimates we combine Theorem 1.1 with the following bounds on $A$ :

$$
A_{0}(1+|x|)^{2-\alpha-\beta} \leq A(x) \leq A_{1}(1+|x|)^{2-\alpha-\beta} .
$$

This double inequality can be proven in all cases $(\mathrm{C} 1)-(\mathrm{C} 4)$ by estimating the solution constructed in (7.2) for $C_{0}=C_{1}=0$,

$$
A(r)=\int_{0}^{r} \frac{s^{1-n}}{b(s)} \int_{0}^{s} a(\rho) \rho^{n-1} d \rho d s,
$$

since the solutions for $(\mathrm{C} 1)-(\mathrm{C} 4)$ are slight modifications of this function. Thus, in (C2) the solution is a product between an angular function (which does not change with the radius) and the above $A(r)$; hence its estimate will not change for large $r$. The functions of (C3) and (C4) have exactly the above form with $a$ and $b$ replaced by explicit expressions which satisfy the same order of growth as $r \rightarrow \infty$. The constants $A_{0}$ and $A_{1}$ may change from case to case, but here we are not interested in obtaining its exact value, even though this is not difficult to do.

One can easily obtain (6.1) from (6.2) by using the bounds of (1.3) and (1.2) together with the following inequalities:

$$
\begin{aligned}
& \frac{C_{0}}{s^{\beta}}<\frac{1}{(1+s)^{\beta}}<\frac{1}{s^{\beta}}, \quad s>0, \\
& \frac{C_{1}}{s^{\alpha}}<\frac{1}{(1+s)^{\alpha}}<\frac{C_{2}}{s^{\alpha}}, \quad s>0,
\end{aligned}
$$

which hold for some $C_{0}, C_{1}<1, C_{2}=1$ if $\alpha$ and $\beta$ are both nonnegative. If $\alpha<0$ simply choose $C_{1}=1, C_{2}>1$. The estimates from (6.1) follow after a basic integration. The energy estimate follows easily by using $(a 2)$.

The weighted $L^{2}$-norm of $u$ requires more work. First, since $\mu>0$, we have by Theorem 1.1 that

$$
\int e^{(\mu-\delta) \frac{A(x)}{t}} a(x) u^{2} d x \leq C_{\delta}\left(\left\|\nabla u_{0}\right\|_{L^{2}}^{2}+\left\|u_{1}\right\|_{L^{2}}^{2}\right) t^{\delta-\mu} .
$$

If $\alpha \leq 0$, the $L^{2}$-estimate in Corollary 1.4 follows from $a(x) \geq a_{0}$. If $\alpha>0$, we use the upper bound on $A(x)$ from (6.1) and property (1.3) of $a(x)$ to show that

$$
\begin{aligned}
a(x) & \geq C(A(x))^{-\frac{\alpha}{2-\alpha-\beta}} \\
& =C t^{-\frac{\alpha}{2-\alpha-\beta}}\left(\frac{A(x)}{t}\right)^{-\frac{\alpha}{2-\alpha-\beta}} \\
& \geq C t^{-\frac{\alpha}{2-\alpha-\beta}} e^{-\delta \frac{A(x)}{t}}
\end{aligned}
$$


with $C>0$, whenever $t$ is sufficiently large. We can complete the proof by substituting this lower bound of $a(x)$ into inequality (6.3).

Proof of Corollary 1.4. The corollary restates the theorem in case (C4) so that we have the bound on $\mu$ which is proved in Section 7 in (7.9).

Proof of Corollary 1.5. The proof is an immediate consequence of Corollary 1.4 and (7.10) from Section 7.

\section{Solutions And Subsolutions to $\operatorname{div}(b(x) \nabla A(x))=a(x)$}

To derive precise decay estimates from Theorem 1.1 we need better knowledge of the function $A$. We will find several cases when we can explicitly construct $A$ that satisfy conditions $(a 1)-(a 3)$.

(C1). Radial coefficients $a$ and $b$. We show here that assumptions (a1)-(a3) are satisfied in the radial case. We begin by finding an explicit solution $A$ for (5.1) with the above properties for radial functions $a$ and $b$. In this case (5.1) becomes

$$
b(r)\left(A_{r r}+\frac{n-1}{r} A_{r}\right)+b_{r} A_{r}=a(r), \quad r=|x| .
$$

To reduce the order, we multiply by $r^{n-1}$ :

$$
\left(r^{n-1} b(r) A_{r}\right)_{r}=r^{n-1} a(r)
$$

so we have after integration that

$$
A_{r}=\frac{r^{1-n}}{b(r)}\left(C_{0}+\int_{r_{0}}^{r} \rho^{n-1} a(\rho) d \rho\right)
$$

We find a solution in the form

$$
A(r)=C_{1}+\int_{0}^{r} \frac{s^{1-n}}{b(s)}\left(C_{0}+\int_{0}^{s} a(\rho) \rho^{n-1} d \rho\right) d s .
$$

By letting $C_{0}=C_{1}=0$ we obtain the solution which satisfies $A(0)=A_{r}(0)=0$.

It is easy to see that $(a 1)$ is satisfied. From (1.3) and (1.2) we can assume that for large values of $r$ we have $a(r) \sim C r^{-\alpha}$ and $b(r) \sim C r^{\beta}$ (in the sequel $C$ is a positive constant that can change from line to line). From (7.1) we deduce that for arbitrary $C_{0}$ we have for large $r$ :

$$
A_{r} \sim C r^{\max \{1-n-\beta, 1-\alpha-\beta\}} \sim C r^{1-\alpha-\beta},
$$

since $\alpha<n$. For $C_{1}$ possibly nonzero we obtain by (7.2) at infinity that $A(r)$ behaves like

$$
A(r) \sim C r^{\max \{0,2-\alpha-\beta\}} .
$$

Note that $\max \{0,2-\alpha-\beta\}=2-\alpha-\beta$. This can be proved by analyzing each of the cases:

- $2 \alpha+\beta \leq 2, \beta<2, \alpha>0$. We have that $\alpha+\beta<2 \alpha+\beta \leq 2$.

- $\alpha \leq 0, \beta<2$. Again, trivial to see since $0<2-\beta \leq 2-\alpha-\beta$.

Hence,

$$
A(r) \sim C r^{2-\alpha-\beta}
$$


Assumption ( $a 2)$ follows immediately. In order to prove ( $a 3)$ we need to estimate the quotient from the definition of $\mu$. Note that for the allowable ranges of exponents $\alpha$ and $\beta$ we have that $2-\alpha-\beta \neq 0$. It follows that

$$
\frac{a(r) A(r)}{b(r)\left[A_{r}(r)\right]^{2}} \sim C \frac{r^{-\alpha} \cdot r^{2-\alpha-\beta}}{r^{\beta} \cdot\left(r^{1-\alpha-\beta}\right)^{2}} \sim C>0 .
$$

Since the rate of growth for the above quantity is always nonnegative, we have that

$$
\mu=\liminf _{r \rightarrow \infty} \frac{a(r) A(r)}{b(r)\left[A_{r}(r)\right]^{2}}>0 .
$$

(C2). Nonradial coefficients - $a$ and $b$ separable. In this case we can also construct a solution to the elliptic equation. Consider the decomposition of the point $x \in \mathbb{R}^{n}$ in the radial components $(r, \omega)$, where $r=|x|$ and $\omega=\frac{x}{r}$ is the projection of $x$ on the $n-1$ dimensional unit sphere. Assume that $a$ and $b$ can be written such that

$$
a(x)=a(r, \omega)=a_{1}(r) \zeta(\omega), \quad b(x)=b(r, \omega)=b_{1}(r) \zeta(\omega)
$$

where the function $\zeta(\omega)$ gives the dependence of $a(x)$ and $b(x)$ on the position of $\omega$ on the unit sphere, and $a_{1}, b_{1}$ are the radial components of $a$, respectively, $b$. Under this assumption we can construct a radial solution $A$ to (5.1) which satisfies $(a 1)-(a 3)$. Define

$$
A(r):=\int_{r_{0}}^{r} \int_{r_{0}}^{s} \frac{s^{1-n}}{b_{1}(s)} a_{1}(\rho) \rho^{n-1} d \rho d s=\int_{r_{0}}^{r} \int_{r_{0}}^{s} \frac{s^{1-n}}{b(s, \omega)} a(\rho, \omega) \rho^{n-1} d \rho d s
$$

where the last equality holds true by (7.3).

For $b(x)=b_{1}(r) \zeta\left(\frac{x}{r}\right)$ we compute

$$
\frac{\partial b}{\partial x_{i}}=\left(b_{1}\right)_{r} \zeta \frac{x_{i}}{r}+b_{1}\left(\frac{\partial \zeta}{\partial x_{i}} \frac{1}{r}-\frac{x_{i}}{r^{3}} \nabla \zeta \cdot x\right)
$$

which yields

$$
\nabla b=\left(b_{1}\right)_{r} \frac{x}{r} \zeta+b_{1} \nabla \zeta\left(\frac{I}{r}-\frac{x \otimes x}{r^{3}}\right) .
$$

We use the above equality and the fact that $A$ is radial to compute

$$
\operatorname{div}(b \nabla A)=\nabla b \cdot \nabla A+b \Delta A=\left(b_{1}\right)_{r} A_{r} \zeta+b \Delta A=\left(b_{1}\right)_{r} A_{r} \zeta+b_{1} \zeta\left(A_{r r}+\frac{n-1}{r} A_{r}\right) .
$$

Hence, equation (5.1) can be reduced to the radial case

$$
\left(b_{1}\right)_{r} A_{r}+b_{1}\left(A_{r r}+\frac{n-1}{r} A_{r}\right)=a_{1} .
$$

These computations were shown in case (C1); hence, we have that $A$ is a solution to (5.1). 
(C3). Arbitrary coefficient $a$ and radial $b$. These assumptions allow us to construct a subsolution. Let $A_{1}$ satisfy

$$
\operatorname{div}\left(b(x) \nabla A_{1}(x)\right)=\frac{a_{1}}{(1+|x|)^{\alpha}},
$$

where $a_{1}$ is given by (1.3). Since $b$ is radial the equations above are equivalent to

$$
b_{r} A_{1_{r}}+b\left(A_{1_{r r}}+\frac{n-1}{r} A_{1_{r}}\right)=\frac{a_{1}}{(1+r)^{\alpha}} ;
$$

hence we can take (see the discussion for the radial case above)

$$
A_{1}(r):=a_{1} \int_{r_{0}}^{r} \int_{r_{0}}^{s} \frac{s^{1-n}}{b(s)} \frac{\rho^{n-1}}{(1+\rho)^{\alpha}} d \rho d s
$$

By (1.3) we have that $A_{1}$ is a subsolution of (5.1). As was remarked earlier, $(\sigma 1)$ $(\sigma 3)$ hold if instead of taking an exact solution to (5.1) we consider the subsolution $A_{1}$. Note that for this construction we used only the bound $a_{1}$ from (1.3).

Remark. This case covers the situation encountered in the main result (Theorem 1.2) of [11, since $b=1$ is radial, so that we can eliminate the radial assumption imposed for $a$. To this end we also use the fact that a subsolution is sufficient to establish the decay rates in terms of $\mu$, where $\mu$ is given by (a3). Note, however, that the explicit decay rate is obtained from the equality $\mu=\frac{n-\alpha}{2-\alpha}$ only under the assumption that the solutions are (almost) radial at infinity.

(C4). Arbitrary coefficients $a$ and $b$. In order to find a subsolution in this case we begin by solving the radial equation where the coefficients $a$ and $b$ are replaced by appropriate radial bounds

$$
\operatorname{div}\left(b_{0}(1+r)^{\beta} \nabla A_{1}(r)\right)=\frac{a_{1}}{(1+r)^{\alpha}} .
$$

We find a solution $A_{1}$ which satisfies

$$
A_{1_{r}}(r)=\frac{r^{1-n}}{b_{0}(1+r)^{\beta}} \int_{r_{0}}^{r} \rho^{n-1} \frac{a_{1}}{(1+\rho)^{\alpha}} d \rho
$$

so that

$$
A_{1}(r)=\int_{r_{0}}^{r} \frac{s^{1-n}}{b_{0}(1+s)^{\beta}} \int_{r_{0}}^{s} \rho^{n-1} \frac{a_{1}}{(1+\rho)^{\alpha}} d \rho d s .
$$

(The computations are the ones presented in the case $a, b$ radial with $a=\frac{a_{1}}{(1+r)^{\alpha}}$ and $b=b_{0}(1+r)^{\beta}$, where we chose the integration constants from (17.2) to be $C_{0}=C_{1}=0$.) Hence, $A_{1}$ satisfies

$$
\left(b_{0}(1+r)^{\beta}\right)_{r} A_{1_{r}}+b_{0}(1+r)^{\beta} \Delta A_{1}=\frac{a_{1}}{(1+r)^{\alpha}} .
$$


With this $A_{1}$ we perform the following computation:

$$
\begin{aligned}
\Delta A_{1} & =\frac{\frac{a_{1}}{(1+r)^{\alpha}}-\left(b_{0}(1+r)^{\beta}\right)_{r} A_{1_{r}}}{b_{0}(1+r)^{\beta}} \\
& =\frac{a_{1}}{b_{0}(1+r)^{\alpha+\beta}}-\frac{\beta r^{1-n}}{b_{0}(1+r)^{\beta+1}} \int_{r_{0}}^{r} \rho^{n-1} \frac{a_{1}}{(1+\rho)^{\alpha}} d \rho \\
& =\frac{a_{1}}{(1+r)^{\alpha}}\left[1-\int_{r_{0}}^{r}\left(\frac{\rho}{r}\right)^{n-1}\left(\frac{1+r}{1+\rho}\right)^{\alpha} \frac{1}{1+r} d \rho\right] .
\end{aligned}
$$

Under the additional assumption

$$
\alpha<n-1
$$

we see that $\Delta A_{1}>0$.

This suffices to prove that $A_{1}$ is a subsolution of the initial elliptic problem, i.e. it satisfies (5.4), whenever

$$
\nabla b \cdot \frac{x}{r} \geq b_{0} \beta(1+r)^{\beta-1}
$$

since we have

$$
\operatorname{div}\left(b(x) \nabla A_{1}(x)\right)=\nabla b \cdot \nabla A_{1}+b \Delta A_{1}=\nabla b \cdot \frac{x}{r} A_{1_{r}}+b \Delta A_{1}
$$

and

$$
b_{0} \beta(1+r)^{\beta-1} A_{1_{r}}+b_{0}(1+r)^{\beta} \Delta A_{1} \geq \frac{a_{1}}{(1+r)^{\alpha}} \geq a(x) .
$$

In the above computation we also used the bounds given by (1.3), (1.2) and the fact that $A_{1_{r}}>0$.

Next we find a bound for the decay rate

$$
\mu=\liminf _{x \rightarrow \infty} \frac{a(x) A_{1}(|x|)}{b(x)\left[A_{1_{r}}(|x|)\right]^{2}}
$$

and show that it is strictly positive.

First note that under (7.6) we have from (7.5) and (7.4) that for large $r$

$$
A_{1_{r}}(r) \sim \frac{a_{1}}{b_{0}(n-\alpha)} r^{1-\alpha-\beta}
$$

and

It then follows that

$$
A_{1}(r) \sim \frac{a_{1}}{b_{0}(n-\alpha)(2-\alpha-\beta)} r^{2-\alpha-\beta}
$$

$$
\mu=\liminf _{x \rightarrow \infty} \frac{n-\alpha}{2-\alpha-\beta} \cdot \frac{b_{0}}{a_{1}} \cdot \frac{a(x)}{b(x)}|x|^{\alpha+\beta} .
$$

From (1.3) and (1.2) we obtain that

$$
\mu \geq \frac{a_{0} b_{0}}{a_{1} b_{1}} \cdot \frac{n-\alpha}{2-\alpha-\beta}>0 .
$$

Note that for $a$ and $b$ which satisfy (1.10) we have $a_{0}=a_{1}=a_{2}, b_{0}=b_{1}=b_{2}$, so then following the above argument we obtain the equality

$$
\mu=\frac{n-\alpha}{2-\alpha-\beta} .
$$




\section{Appendix A. Proof of the energy identity}

Upon multiplication of (3.2) by $\eta u$ and integration over $\mathbb{R}^{n}$ we obtain

$$
\begin{aligned}
& \frac{d}{d t} \int\left(\eta \hat{u}_{t} \hat{u}+\eta \hat{a}_{1} \frac{\hat{u}^{2}}{2}\right) d x-\int\left(\eta_{t} \hat{u}_{t} \hat{u}+\eta \hat{u}_{t}^{2}\right) d x+\int \nabla \eta \cdot \nabla \hat{u} \hat{b}_{1} \hat{u} d x \\
& +\int\left(\eta \hat{u} \nabla \hat{b}_{1} \cdot \nabla \hat{u}+\eta \hat{b}_{1}|\nabla \hat{u}|^{2}+\eta \hat{a}_{2} \hat{u}^{2}\right) d x+\int\left[\operatorname{div}\left(\eta \hat{b}_{2}\right)-\left(\eta \hat{a}_{1}\right)_{t}\right] \frac{\hat{u}^{2}}{2} d x=0 .
\end{aligned}
$$

The equality can be rewritten in the following form after integration by parts:

$$
\begin{aligned}
& \frac{d}{d t} \int\left(\eta \hat{u}_{t} \hat{u}+\eta \hat{a}_{1} \frac{\hat{u}^{2}}{2}\right) d x-\frac{1}{2} \int\left[\left(\eta_{t} \hat{u}^{2}\right)_{t}+2 \eta \hat{u}_{t}^{2}\right] d x \\
& \quad+\int\left[\operatorname{div}\left(\hat{u}^{2} \hat{b}_{1} \nabla \eta\right)+\eta \hat{u} \nabla b_{1} \cdot \nabla \hat{u}+\eta \hat{b}_{1}|\nabla u|^{2}\right] d x \\
& \quad+\int \frac{\hat{u}^{2}}{2}\left[2 \eta \hat{a}_{2}-\eta_{t t}-\nabla \hat{b}_{1} \cdot \nabla \eta-\hat{b}_{1} \Delta \eta+\operatorname{div}\left(\eta \hat{b}_{2}\right)-\left(\eta \hat{a}_{1}\right)_{t}\right] d x=0
\end{aligned}
$$

Similarly, by using the multiplier $\theta u_{t}$ for (3.2) we obtain

$$
\int\left[\theta\left(\frac{\hat{u}_{t}^{2}}{2}\right)_{t}-\theta \hat{b}_{1} \Delta \hat{u} \hat{u}_{t}-\theta \hat{b}_{2} \cdot \nabla \hat{u} \hat{u}_{t}+\theta \hat{a}_{1} \hat{u}_{t}^{2}+\theta \hat{a}_{2}\left(\frac{\hat{u}^{2}}{2}\right)_{t}\right] d x=0 .
$$

Integration by parts and a rearrangement of terms gives

$$
\begin{aligned}
& \frac{1}{2} \frac{d}{d t} \int\left(\theta \hat{u}_{t}^{2}+\hat{b}_{1}|\nabla \hat{u}|^{2}+\theta \hat{a}_{2} \hat{u}^{2}\right) d x-\frac{1}{2} \int\left(\theta_{t} \hat{u}_{t}^{2}+\left(\theta \hat{b}_{1}\right)_{t}|\nabla u|^{2}\right) d x \\
+ & \int\left[\hat{u}_{t} \hat{b}_{1} \nabla \hat{u} \cdot \nabla \theta+\hat{u}_{t} \theta \nabla \hat{u} \cdot \nabla \hat{b}_{1}-\theta \hat{u}_{t} \hat{b}_{2} \cdot \nabla \hat{u}+\theta \hat{a}_{1} \hat{u}_{t}^{2}-\left(\theta \hat{a}_{2}\right)_{t} \frac{\hat{u}^{2}}{2}\right] d x=0 .
\end{aligned}
$$

By adding (A.1) and (A.2) and combining similar terms we obtain

$$
\begin{aligned}
& \frac{1}{2} \frac{d}{d t} \int \theta\left(\hat{u}_{t}^{2}+\hat{b}_{1}|\nabla \hat{u}|^{2}\right)+2 \eta \hat{u}_{t} u+\left(\theta \hat{a}_{2}-\eta_{t}+\eta \hat{a}_{1}\right) \hat{u}^{2} d x \\
+ & \int\left(\frac{-\theta_{t}}{2}+\theta \hat{a}_{1}-\eta\right) \hat{u}_{t}^{2}+\left(\eta \hat{b}_{1}-\frac{\left(\hat{b}_{1} \theta\right)_{t}}{2}\right)|\nabla \hat{u}|^{2} d x \\
+ & \int \hat{u}_{t} \nabla \hat{u} \cdot\left(\hat{b}_{1} \nabla \theta+\theta \nabla \hat{b}_{1}-\theta \hat{b}_{2}\right) d x \\
+ & \int \frac{\hat{u}^{2}}{2}\left(\eta_{t t}-\hat{b}_{1} \Delta \eta+2 \eta \hat{a}_{2}-\left(\eta \hat{a}_{1}\right)_{t}+\operatorname{div}\left(\eta \hat{b}_{2}\right)-\left(\theta \hat{a}_{2}\right)_{t}-\nabla \hat{b}_{1} \cdot \nabla \eta\right) d x \\
+ & \int \eta \hat{u} \nabla \hat{b}_{1} \cdot \nabla \hat{u} d x=0 .
\end{aligned}
$$

Since $\hat{u} \nabla \hat{u}=\frac{1}{2} \nabla\left(\hat{u}^{2}\right)$ we can write, after an integration by parts,

$$
\int\left[-\frac{\hat{u}^{2}}{2} \operatorname{div}\left(\hat{b}_{1} \nabla \eta\right)+\eta \hat{u} \nabla \hat{b}_{1} \cdot \nabla \hat{u}\right] d x=-\int \frac{\hat{u}^{2}}{2} \operatorname{div}\left[\nabla\left(\hat{b}_{1} \eta\right)\right] d x .
$$

This completes the proof of the energy identity in Proposition 3.1. 


\section{REFERENCES}

[1] Barbu, V. Nonlinear semigroups and differential equations in Banach spaces, Editura Academiei, Bucuresti Romania and Noordhoff International Publishing, Leyden, the Netherlands (1976). MR0390843 (52:11666)

[2] Evans, L.C., Partial Differential Equations, Graduate Studies in Mathematics, AMS, vol. 19 (1998). MR1625845 (99e:35001)

[3] Feng, Shao Ji; Feng, De Xing, Nonlinear internal damping of wave equations with variable coefficients, Acta Math. Sin. (Engl. Ser.) 20 (2004), no. 6, 1057-1072. MR2130371 (2005m:35190)

[4] Ikawa, Mitsuru, Hyperbolic partial differential equations and wave phenomena, American Mathematical Society, Providence R.I. (2000). MR.1756774 (2001j:35176)

[5] Ikehata, R., Local energy decay for linear wave equations with variable coefficients, J. Math. Anal. Appl. 306 (2005), no. 1, 330-348. MR2132904 (2006a:35187)

[6] Milani, Albert; Yang, Han On the diffusion phenomenon of quasilinear hyperbolic waves in low space dimensions, Bull. Sci. Math. 124 (2000), 415-433. MR1781556 (2001f:35271)

[7] Morawetz, C., The decay of solutions of the exterior initial-boundary problem for the wave equation, Comm. Pure Appl. Math. Sci. 14 (1961), 561-568. MR0132908 (24:A2744)

[8] Narazaki, T. $L^{p}-L^{q}$ estimates for damped wave equations and their applications to semi-linear problem, J. Math. Soc. Japan 56 (2004), 585-626. MR2048476 (2005a:35206)

[9] Reissig, M., $L^{p}-L^{q}$ decay estimates for wave equations with time-dependent coefficients, J. Nonlinear Math. Phys. 11 (2004), no. 4, 534-548. MR2098544 (2006b:35232)

[10] Reissig, M. and Wirth, J., $L^{p}-L^{q}$ estimates for wave equations with monotone timedependent dissipation, Proceedings of the RIMS Symposium on Mathematical Models of Phenomena and Evolution Equations (to appear).

[11] Todorova, G; Yordanov, B., Weighted $L^{2}$ Estimates for Dissipative Wave Equations with Variable Coefficients (to appear).

[12] Todorova, G; Yordanov, B., Nonlinear Dissipative Wave Equations with Potential, Control methods in PDE-dynamical systems, Contemp. Math., 426, Amer. Math. Soc., Providence, RI (2007), 317-337. MR 2311533

[13] Wirth, J., Solution representations for a wave equation with weak dissipation, Math. Methods Appl. Sci. 27 (2004), no. 1, 101-124. MR2023397 (2004k:35040)

[14] Wirth, J., Wave equations with time-dependent dissipation. I. Non-effective dissipation, J. Diff. Eq. 222 (2006), 487-514. MR2208294 (2007d:35171)

[15] Wirth, J., Wave equations with time-dependent dissipation. II. Effective dissipation, J. Differential Equations 232 (2007), no. 1, 74-103. MR2281190 (2007k:35293)

Department of Mathematics and Statistics, University of Nebraska-Lincoln, LinCOLN, NEBRASKa 68588

E-mail address: pradu@math.unl.edu

Department of Mathematics, University of Tennessee, Knoxville, Knoxville, TenNESSEE 37996

E-mail address: todorova@math.utk.edu

Department of Mathematics, University of Tennessee, Knoxville, Knoxville, TenNESSEE 37996

E-mail address: yordanov@math.utk.edu 